\title{
Transcriptome analysis of the adult human Klinefelter testis and cellularity-matched controls reveals disturbed differentiation of Sertoli- and Leydig cells
}

Sofia Boeg Winge ${ }^{1}$, Marlene Danner Dalgaard ${ }^{1,2}$, Kirstine G Belling ${ }^{3}$, Jacob Malte Jensen ${ }^{4}$, John Erik Nielsen', Lise Aksglaede1, Mikkel Heide Schierup ${ }^{4}$, Søren Brunak ${ }^{3}$, Niels Erik Skakkebæk', Anders Juul',

Ewa Rajpert-De Meyts (iD ${ }^{1}$ and Kristian Almstrup ${ }^{1}$

\begin{abstract}
The most common human sex chromosomal disorder is Klinefelter syndrome (KS; 47,XXY). Adult patients with KS display a diverse phenotype but are nearly always infertile, due to testicular degeneration at puberty. To identify mechanisms causing the selective destruction of the seminiferous epithelium, we performed RNA-sequencing of 24 fixed paraffin-embedded testicular tissue samples. Analysis of informative transcriptomes revealed 235 differentially expressed transcripts (DETs) in the adult KS testis showing enrichment of long non-coding RNAs, but surprisingly not of X-chromosomal transcripts. Comparison to $46, X Y$ samples with complete spermatogenesis and Sertoli cell-onlysyndrome allowed prediction of the cellular origin of 71 of the DETs. DACH2 and FAM9A were validated by immunohistochemistry and found to mark apparently undifferentiated somatic cell populations in the KS testes. Moreover, transcriptomes from fetal, pre-pubertal, and adult KS testes showed a limited overlap, indicating that different mechanisms are likely to operate at each developmental stage. Based on our data, we propose that testicular degeneration in men with KS is a consequence of germ cells loss initiated during early development in combination with disturbed maturation of Sertoli- and Leydig cells.
\end{abstract}

\section{Introduction}

Klinefelter syndrome (KS) is caused by the presence of at least one additional $\mathrm{X}$-chromosome in men. KS is the most common sex chromosomal disorder with an estimated prevalence of 1 in 660 newborn boys, but only one fourth of the expected cases are diagnosed throughout life ${ }^{1}$. There is a huge variation in the phenotype of men with KS. In adulthood, the classical symptoms are tall stature, gynecomastia, reduced virilization, hypergonadotropic

\footnotetext{
Correspondence: Kristian Almstrup (kristian.almstrup@regionh.dk)

${ }^{1}$ Department of Growth and Reproduction, Copenhagen University Hospital

(Rigshospitalet), Copenhagen, Denmark

${ }^{2}$ DTU Multi-Assay Core, DTU Bioinformatics, Technical University of Denmark, Kongens Lyngby, Denmark

Full list of author information is available at the end of the article.

Edited by D. Aberdam
}

hypogonadism, and small testes ${ }^{2-5}$. Many men with KS, however, first discover their condition when they want to have a child as the most pervasive symptom is azoospermia or, rarely, severe oligozoospermia.

The histology of a typical testis from an adult KS man shows absence of germ cells, high amounts of degenerated, hyalinized tubules, and Leydig cell hyperplasia ${ }^{2,6}$. In some men with KS, focal spermatogenesis is nevertheless observed and is considered the result of elimination of the supernumerary X-chromosome in a subset of spermatogonia $^{7-10}$. Presence of focal spermatogenesis opens the possibility to recover sperm in the ejaculate or to perform surgical microdissection of the seminiferous tubules and isolate areas with focal spermatogenesis (micro-TESE procedures) that subsequently can be used for 
intracytoplasmic sperm injection. This allows such patients to become fathers of children with apparently normal karyotype (reviewed in refs. ${ }^{11}$ ).

The molecular mechanisms responsible for the KS phenotype are poorly understood. Best described is the increased dosage of the X-chromosomal SHOX gene, which accounts at least in part for the tall stature observed in men with $\mathrm{KS}^{12}$. Recently, several studies have reported transcriptome analysis of peripheral blood samples ${ }^{13-15}$ and of cultured lymphocytes isolated from blood ${ }^{16}$ from KS patients compared to controls. The only overlap between the transcriptome profiles was upregulation of the long non-coding RNA (lncRNA) XIST ${ }^{13-16}$. Upregulation of XIST is expected and can be used as a quality control, as XIST is involved in X-chromosome inactivation $(\mathrm{XCI})^{17,18}$. Only 12 transcripts were differentially expressed in more than one study ${ }^{13,15,16}$, highlighting the diverse phenotype.

Pre-pubertal testicular development in KS has been described as similar to boys with normal karyotypes, albeit with smaller testis volume and reduced germ cell numbers $^{6,19}$. However, our recent study indicated that despite the supposedly normal pre-pubertal testicular development, the fetal KS testis displays impairment of fetal gonocyte differentiation resulting in loss of prespermatogonia ${ }^{20}$. This effect is most likely caused by the extra X-chromosome because the fetal KS testis transcriptome revealed enrichment of X-chromosomal transcripts as well as $\operatorname{lncRNAs}{ }^{20}$. Later on, initiation of spermatogenesis at pubertal onset fails in the KS testis and results in testicular degeneration ${ }^{6,19}$.

Gene expression analysis of human KS testes is challenging, as available material is scarce because testicular biopsies are not performed in the routine clinical workup. But three recent studies used leftover testis tissue from TESE and micro-TESE. In one study, expression of metabolic components was examined in six KS and six controls with azoospermia but conserved spermatogenesis $^{21}$. In another study, D'Aurora et al. performed microarray analysis of bilateral testicular biopsies from six non-mosaic KS patients with absence of germ cells compared to a pool of three biopsies from patients with obstructive azoospermia but complete spermatogenesis ${ }^{22}$. While the material used in the two studies is unique and very precious, the huge cellularity differences are expected to drive most of the changes in the transcriptome and mask transcripts involved in the actual testicular phenotype of KS. In another study by D'Aurora et al., testicular biopsies from three atypical adult KS patients with focal spermatogenesis were compared to the controls used in their former study ${ }^{23}$. The cellularity differences were thereby less pronounced but the transcriptome now reflected the atypical situation of KS testis with focal spermatogenesis.
To describe the adult KS testicular transcriptome and to account for cellularity differences, we performed RNAsequencing of fixed, paraffin-embedded testicular biopsies from adult KS patients and cellularity-matched controls (CMC), which had Sertoli-cell-only (SCO) pattern and Leydig cell hyperplasia. The cellular origin of the differentially expressed transcripts (DETs) was determined by including samples with complete spermatogenesis. Moreover, to describe developmental changes in the testicular KS transcriptome, we included testis samples from pre-pubertal KS boys along with our published data on the fetal KS testis.

\section{Results}

\section{The adult KS testicular transcriptome}

We performed RNA-sequencing of 15 fixed, paraffinembedded testis samples, and the average reads per sample was 2.8 million (Table 1). The RNA quality was compromised and we, therefore, performed a thorough quality control based on MDS plots, heatmaps and expression of XIST in the KS samples (Fig. 1a and Supplementary Fig. S1). Based on this, three KS samples and three CMC were used in the subsequent analyses (Fig. 1a).

Using an un-adjusted $p$-value $<0.01,235$ DETs were identified, with 153 being upregulated and 82 being downregulated in KS compared to CMC (Fig. 1b, c, and Supplementary Tables S1 and S2). The most significant DET was XIST, as expected. Apart from XIST, eight Xchromosomal DETs were upregulated (Fig. $1 \mathrm{~b}$ and Supplementary Table S1); three of these, POU3F4, PNMA5, and $A L P N$, have previously been reported to undergo $\mathrm{XCI}^{24}$. The remaining upregulated transcripts have no or discordant data regarding XCI status. Four Xchromosomal transcripts were downregulated, one expressed from PAR1: ASMTL-AS1, one previously reported to undergo XCI: $L O N R F 3$, and two with no or discordant data regarding XCI (Fig. 1b and Supplementary Table S2).

\section{Protein expression of DACH2 and FAM9A}

To validate our findings, we selected two upregulated $\mathrm{X}$ chromosomal candidates, DACH2 and FAM9A based on availability of antibodies and supportive literature.

Immunohistochemical staining showed that DACH2 was expressed in the nuclei. In testes with complete spermatogenesis, DACH2 showed strongest intensity in the wall of blood vessels, medium-strong intensity in primary spermatocytes, and faint-negative staining in Sertoli cells (Supplementary Fig. S2). In testes from adult men with $\mathrm{KS}(n=2)$, DACH2 was expressed in a subset of Sertoli cell nuclei of type A (mature) and type B (immature) SCO tubules (identified according to ref. ${ }^{25}$ ) (Fig. 2a, b). Sertoli cell nuclei showed only faint $\mathrm{DACH} 2$ staining in CMC biopsies $(n=3)$ (Fig. 2c, d), which confirmed 
Table 1 Samples used for RNA-sequencing

\begin{tabular}{|c|c|c|c|c|c|c|c|}
\hline ID & Group & Age & Karyotype (blood) & Fixative & Block age & Library size & Included in final analysis/ reason for exclusion \\
\hline aKS1 & Adult KS & $21 \mathrm{y}, 7 \mathrm{~m}$ & $47, X X Y$ & GR-fix & $1 y$ & $7,413,356$ & Yes \\
\hline aKS2 & Adult KS & $27 y, 7 m$ & $47, X X Y$ & Bouin & $14 y$ & $1,693,175$ & Yes \\
\hline aKS3 & Adult KS & $28 y, 7 m$ & $47, X X Y$ & GR-fix & $4 y$ & $2,199,570$ & Yes \\
\hline $\mathrm{aKS} 4^{\mathrm{a}}$ & Adult KS & $30 y, 7 m$ & & Stieve & $42 y$ & 812,877 & No XIST expression \\
\hline aKS5 & Adult KS & $40 y, 2 m$ & $47, X X Y$ & Stieve & $16 y$ & 177,280 & Low library size and MDS plot \\
\hline aKS6 & Adult KS & $21 y, 3 m$ & $48, X X Y Y, 47, X X Y$ & Stieve & $40 y$ & 132,405 & Low library size and MDS plot \\
\hline $\mathrm{aKS} 7^{\mathrm{a}}$ & Adult KS & $27 \mathrm{y}, 11 \mathrm{~m}$ & & Stieve & $48 y$ & 541,108 & MDS plot \\
\hline $\mathrm{aKS}^{\mathrm{a}}$ & Adult KS & $28 \mathrm{y}, 6 \mathrm{~m}$ & & Stieve & $48 y$ & 123,653 & No XIST expression \\
\hline aKS9 & Adult KS, GCs & $21 \mathrm{y}, 7 \mathrm{~m}$ & $47, X X Y$ & GR-fix & $1 y$ & $8,233,082$ & Same patient as aKS1 \\
\hline aKS10 & Adult KS, GCs & $30 y, 0 m$ & $47, X X Y$ & Stieve & $14 y$ & 188,313 & Low library size and MDS plot \\
\hline $\mathrm{aCMC1}$ & Adult CMC & $32 \mathrm{y}, 0 \mathrm{~m}$ & $46, X Y$ & GR-fix & $6 y$ & $4,480,564$ & Yes \\
\hline $\mathrm{aCMC2}$ & Adult CMC & $32 y, 7 m$ & Two SHOX copies & GR-fix & $4 y$ & $7,868,360$ & Yes \\
\hline $\mathrm{aCMC3}$ & Adult CMC & $33 \mathrm{y}, 8 \mathrm{~m}$ & $46, X Y$ & GR-fix & $7 y$ & $2,044,132$ & Yes \\
\hline $\mathrm{aCMC4}$ & Adult CMC & $39 y, 8 m$ & $46, X Y$ & GR-fix & $5 y$ & $3,113,958$ & Balance with number of KS samples \\
\hline $\mathrm{aCMC5}$ & Adult CMC & $42 y, 2 m$ & $46, X Y$ & GR-fix & $7 y$ & $2,443,579$ & Balance with number of KS samples \\
\hline aSCO1 & Adult SCO & $29 y, 6 m$ & $46, X Y$ & GR-fix & $3 y$ & $3,064,063$ & \\
\hline aSCO2 & Adult SCO & $31 \mathrm{y}, 7 \mathrm{~m}$ & $46, X Y$ & GR-fix & $7 y$ & $2,317,076$ & \\
\hline aSCO3 & Adult SCO & $33 y, 3 \mathrm{~m}$ & $46, X Y$ & GR-fix & $6 y$ & $7,157,424$ & \\
\hline aSCO4 & Adult SCO & $35 y, 4 m$ & $46, X Y$ & GR-fix & $6 y$ & $3,897,228$ & \\
\hline asco5 & Adult SCO & $43 y, 5 m$ & $46, X Y$ & GR-fix & $5 y$ & 634,570 & Low library size and MDS plot \\
\hline aNorm $1^{b}$ & Adult NT & $30 y, 2 m$ & $46, X Y^{d}$ & GR-fix & $4 y$ & $4,635,540$ & Yes \\
\hline aNorm $2^{c}$ & Adult NT & $31 \mathrm{y}, 3 \mathrm{~m}$ & $46, X Y^{e}$ & GR-fix & $6 y$ & $3,100,541$ & Yes \\
\hline aNorm $3^{c}$ & Adult NT & $35 y, 9 m$ & $46, X Y^{e}$ & GR-fix & $6 y$ & $1,213,352$ & Yes \\
\hline aNorm4 ${ }^{b}$ & Adult NT & $39 y, 9 m$ & No karyotype ${ }^{d}$ & GR-fix & $5 y$ & 373,341 & Yes \\
\hline $\mathrm{pKS}^{\mathrm{a}, \mathrm{d}}$ & Pre-pubertal KS & $9 y, 6 \mathrm{~m}$ & & Stieve & $39 y$ & 442,161 & Yes \\
\hline pKS2 $2^{\mathrm{a}, \mathrm{d}}$ & Pre-pubertal KS & $9 y, 6 \mathrm{~m}$ & & Stieve & $35 y$ & 481,952 & Yes \\
\hline pKS3 $3^{\mathrm{a}, \mathrm{d}}$ & Pre-pubertal KS & $11 \mathrm{y}, 0 \mathrm{~m}$ & & Cleland & $32 y$ & 404,698 & Yes \\
\hline pKS4 $4^{\mathrm{a}, \mathrm{d}}$ & Pre-pubertal KS & $14 \mathrm{y}, 1 \mathrm{~m}$ & & Stieve & $37 y$ & 231,918 & Yes \\
\hline $\mathrm{pKS} 5^{\mathrm{a}, \mathrm{d}}$ & Pre-pubertal KS & $14 y, 9 m$ & & Stieve & $39 y$ & $1,120,193$ & No XIST expression \\
\hline pNorm $1^{\mathrm{e}}$ & Pre-pubertal NT & $7 y, 5 \mathrm{~m}$ & & Stieve & $21 y$ & 671,897 & Yes \\
\hline pNorm $2^{f}$ & Pre-pubertal NT & $7 y, 9 m$ & & Cleland & $30 y$ & $1,026,282$ & Yes \\
\hline pNorm $3^{f}$ & Pre-pubertal NT & $10 y, 2 \mathrm{~m}$ & & Cleland & $30 y$ & 392,993 & Yes \\
\hline pNorm4 ${ }^{f}$ & Pre-pubertal NT & $10 y, 10 \mathrm{~m}$ & & Stieve & $35 y$ & 268,552 & Yes \\
\hline
\end{tabular}

KS Klinefelter syndrome, GCs germ cells, SCO Sertoli cell-only, NT normal testis (biopsy with complete spermatogenesis), GR-fix modified Stieve's fixative, y years, $m$ months

${ }^{a}$ Known KS patient, no karyotype available

${ }^{\mathrm{b}}$ Testis cancer patient

'Man from infertile couple

${ }^{d}$ Biopsy taken due to suspicion of urological problems

eAcute lymphocytic leukemia patient

${ }^{f}$ Autopsy 


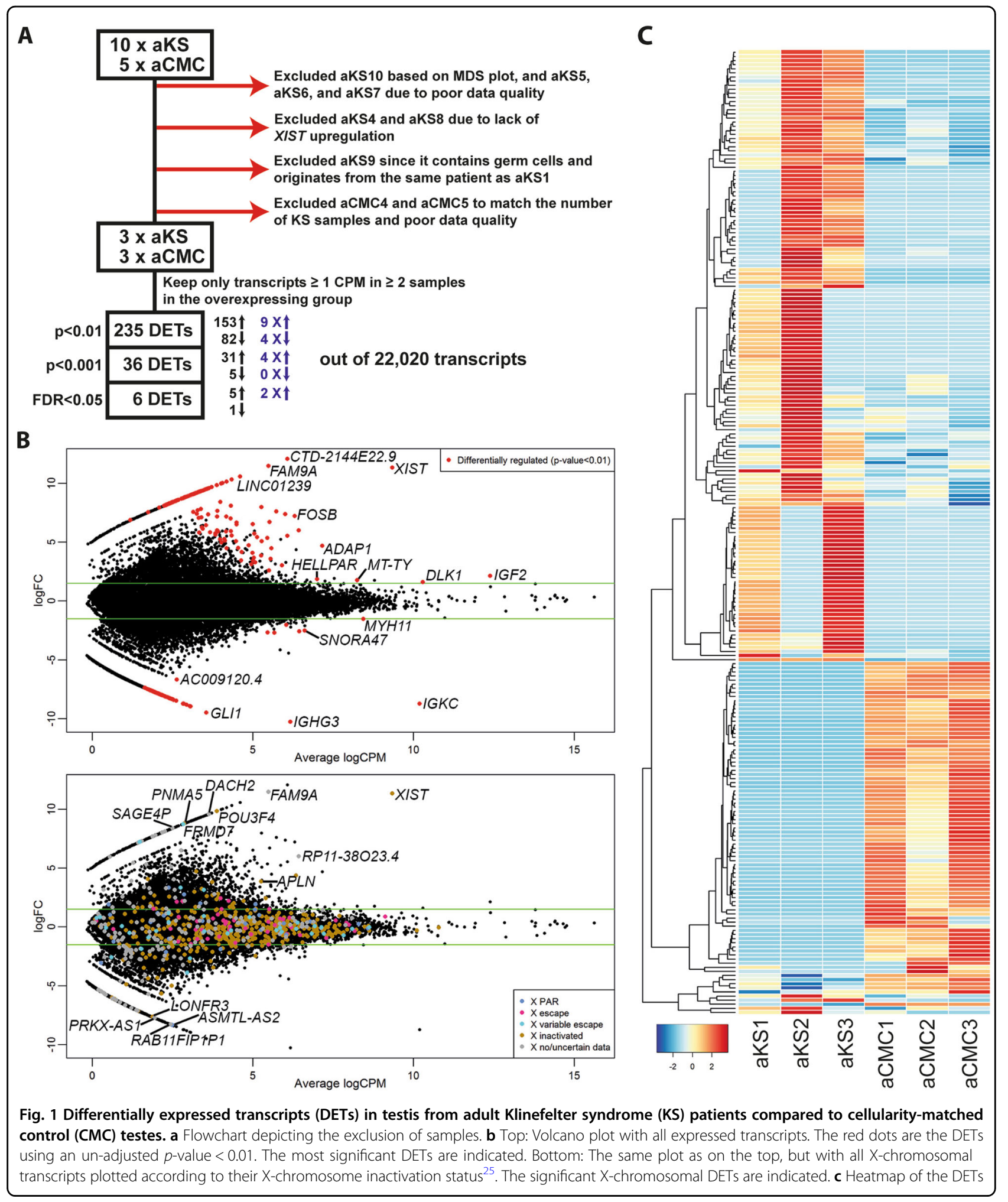

upregulation of DACH2. To test whether DACH2 expression depended on the Sertoli cell maturation stage, an adjacent section was stained with $\mathrm{AMH}$, a marker of immature Sertoli cells. In Sertoli cells, AMH was expressed in the cytoplasm with varying intensity, with the majority of DACH2-positive Sertoli cells also positive for AMH (Supplementary Fig. S3). However, a subset of AMH-positive Sertoli cells were negative for $\mathrm{DACH} 2$, and 


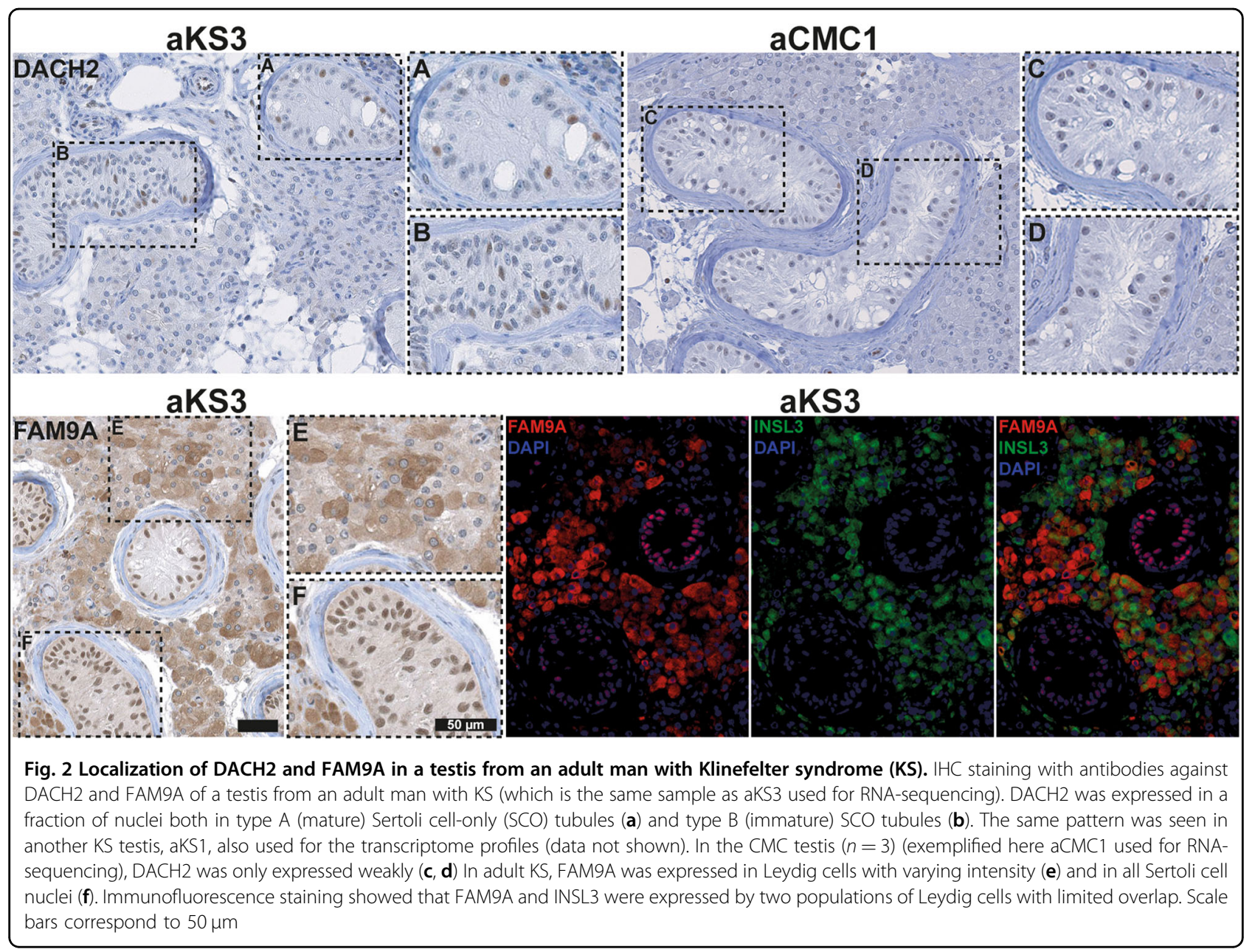

a small proportion of the DACH2-positive Sertoli cells were negative for AMH (Supplementary Fig. S3).

In a fetal testis aged gestational week $(\mathrm{Gw}) 10$, a large fraction of the Sertoli cells indeed showed expression of DACH2 (Supplementary Fig. S2).

In testes with complete spermatogenesis, FAM9A showed strongest intensity in the cell membrane of spermatocytes and spermatids, moderate-strong intensity in Sertoli cell nuclei and faint-strong staining in Leydig cell cytoplasm (Supplementary Fig. S2). In the testis from an adult KS patient, FAM9A was expressed in the cytoplasm of Leydig cells with varying intensity and in the nuclei of all Sertoli cells (Fig. 2e, f), and the same pattern was seen in CMC testes $(n=3)$ (data not shown). A double fluorescent staining with FAM9A and insulin-like 3 (INSL3, a marker of mature Leydig cells ${ }^{26}$ ) showed that FAM9A and INSL3 were only partially overlapping with distinct FAM9A-positive/INSL3-negative and FAM9Anegative/INSL3-positive populations visible (Fig. 2), indicating that FAM9A is expressed predominantly in immature Leydig cells and during the transition phase into mature Leydig cells. In a fetal testis aged Gw10, a fraction of interstitial cells (most probably fetal Leydig cells) were positive for FAM9A (Supplementary Fig. S2).

\section{Enrichment analyses of the KS DETs}

Next, we compared DETs from previous studies of KS patients $^{13-16,22,23}$, and found an overlap of three DETs with the transcriptome profiles from blood and nine DETs from the studies on testes (Fig. 3a, b). Overlapping DETs included FOSB and DLK1 (Supplementary Table S3).

We noticed that many of the DETs were ncRNAs (Supplementary Tables S1 and S2), and we, therefore, analyzed for enrichment of transcript biotypes classified by the GENCODE annotation ${ }^{27}$. We found significant ( $p$ value $=0.0018$ ) enrichment of long, intergenic ncRNAs (lincRNAs) among the upregulated transcripts. Also, protein-coding transcripts among the upregulated ( $p$ value $=0.0058)$ and antisense transcripts among the downregulated transcripts were significantly enriched ( $p$ value $=3.3 \mathrm{E}-5)($ Fig. 4a and Supplementary Table S4).

To gain insight into the potential function of the DETs, we searched for enrichment of particular gene ontologies (GO). Among the most interesting results were those 


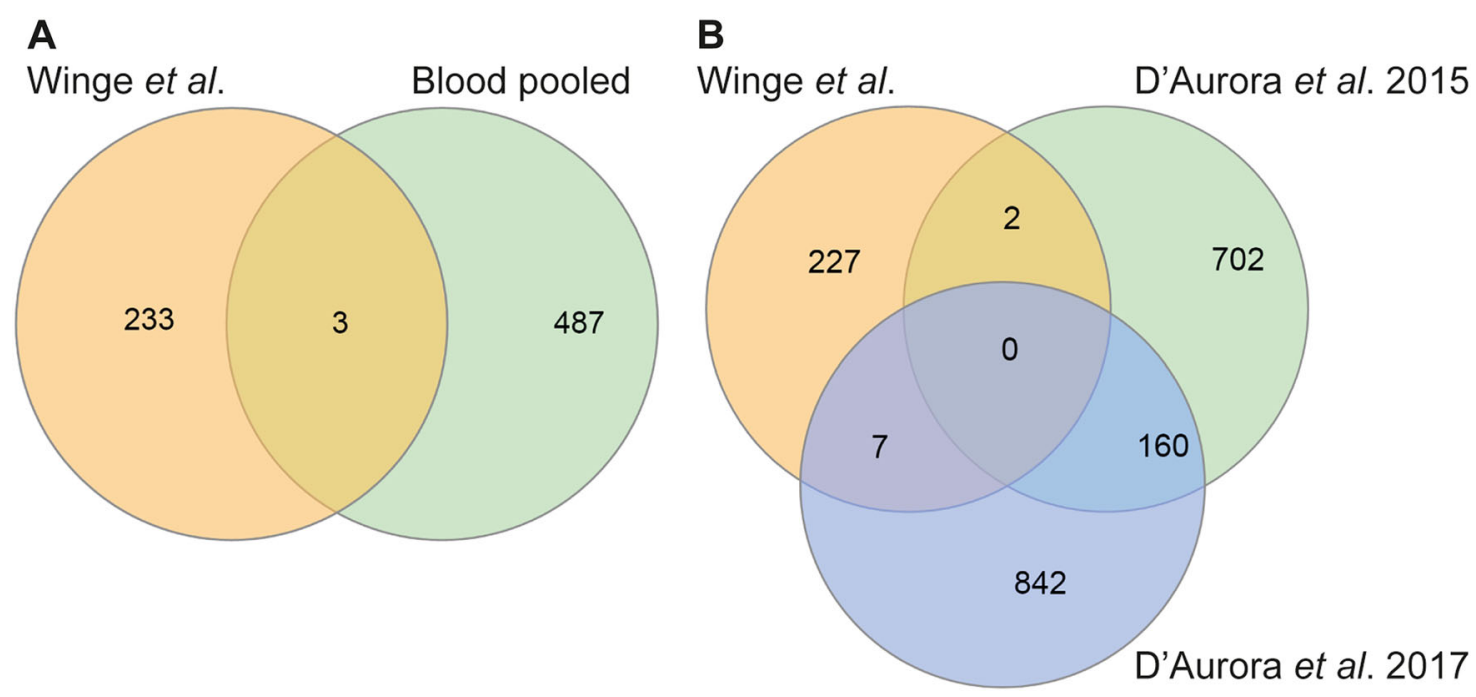

Fig. 3 Overlap of the differentially expressed transcripts (DETs) with previous studies. Overlap with previous studies performed on blood ${ }^{14-17}$ (a) and on testis tissue ${ }^{23,24}$ (b). The overlapping transcripts can be seen in Supplementary Table S3

involved in reproduction ("reproductive process", "reproduction", and "multi-organism reproductive process"), development ("multi-cellular organism development", "single-organism developmental process", "animal organ development", "system development", and "developmental process"), as well as "response to hormone" (Fig. 4b).

\section{Cellular origin of the KS DETs}

In order to identify the potential cellular origin of the 235 DETs, we used a comparative approach, and profiled the transcriptome of five adult testis biopsies with SCO and normal amounts of Leydig cells, and four adult testis biopsies with full spermatogenesis and normal amounts of Leydig cells (these samples are termed Norm) (Table 1 and Supplementary Fig. S4). We excluded one SCO sample due to poor quality (data not shown).

First, we compared the expression of known cell typespecific transcripts in the four groups (Fig. 5a). As expected, $\mathrm{KS}$ and $\mathrm{CMC}$ specimens displayed relative upregulation of Leydig cell transcripts compared to SCO samples, which had a higher level than the Norm samples. The same tendency was seen for peritubular/blood vessel transcripts. The levels of Sertoli cell transcripts were similar among KS, CMC, and SCO, but lower in the Norm samples. And as expected, the levels of germ cell transcripts were highest in Norm samples (Fig. 5a).

We also evaluated the cellularity of each sample by measuring the area of the cellular compartments (Supplementary Fig. S4) and when calculating the average cellularity, we could predict the cellular origin of the DETs for each comparison (Figs. 5b and $6 a)$.
We compared the DETs with those of all of the group comparisons, and thereby could get an idea about the cellular origin of the individual transcripts. There was some overlap between the comparisons, allowing prediction of cellular origin of 71 out of the 235 DETs (Fig. 6a, b). Twenty-six were upregulated in KS and the remaining 45 were downregulated. We were not able to predict the cellular origin of 127 of the upregulated transcripts and 37 of the downregulated transcripts, which could be because the transcripts are expressed in many different cell types or that they are only expressed in the KS testis.

\section{Overlap between DETs in the KS testis throughout development}

In our previous study ${ }^{20}$, we identified 211 DETs in the fetal KS testis compared to controls. To investigate changes of the KS testis transcriptome throughout development, we performed transcriptome analysis of testis samples from pre-pubertal KS boys and agematched controls (Supplementary Fig. S5). The data quality was severely compromised with on average 490,000 reads per sample (Table 1). We did sanity checks, and one of the pre-pubertal KS samples did not have expression of XIST and was excluded (Supplementary Fig. S6). We identified 181 DETs, with 139 being downregulated (Supplementary Fig. S7 and Supplementary Tables S5 and S6).

When we compared the testicular transcriptome profiles at the three different developmental time points in an MDS plot, each age group clustered separately, rather than clustering according to the karyotype (Fig. 7a). Only seven transcripts overlapped between the three developmental time points (Fig. 7b, c, and Supplementary 
A

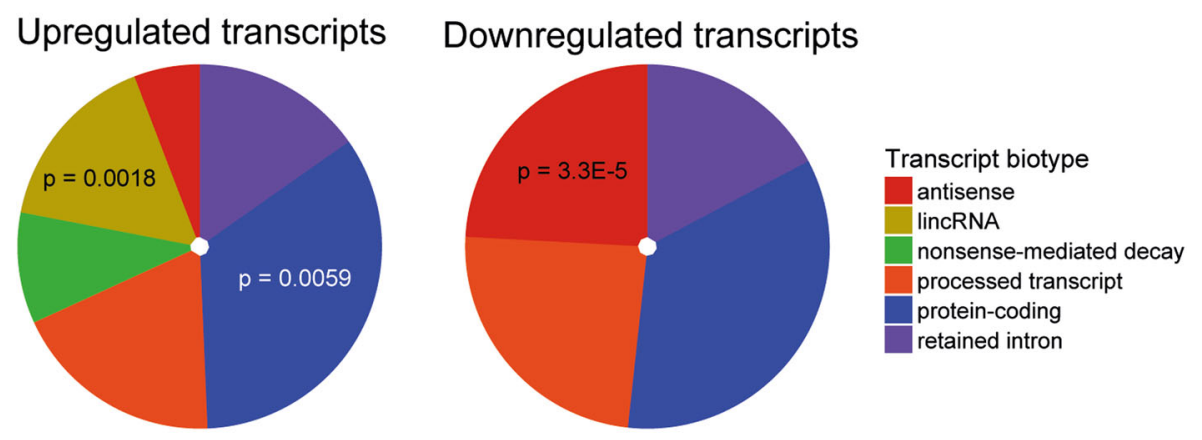

B

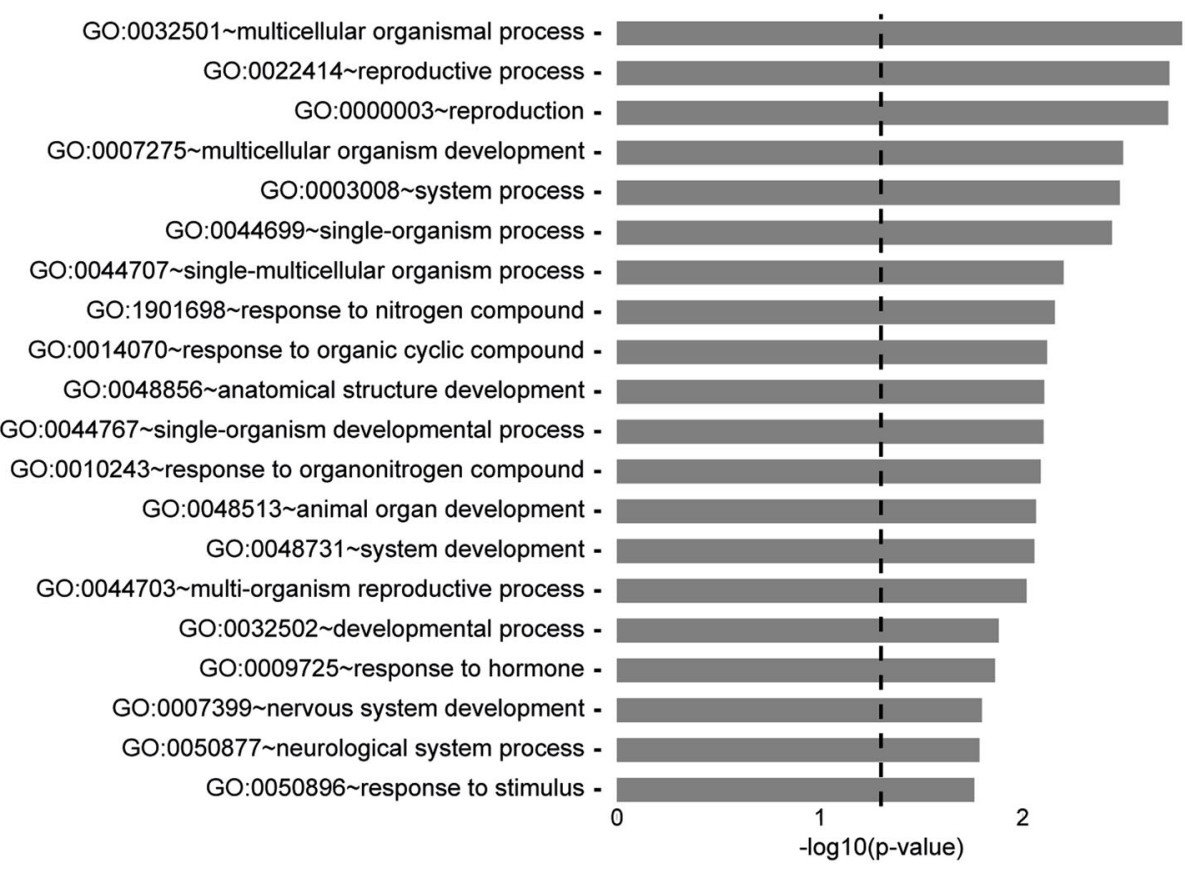

Fig. 4 Enrichment analyses of the differentially expressed transcripts (DETs). a Distribution in RNA biotype among the upregulated (left) and downregulated (right) transcripts. The significant biotypes are indicated with $p$-values. For RNA biotypes of each individual DET, see Supplementary Tables S1 and S2, and for description of RNA biotypes, see Supplementary Table S4. b Significantly overrepresented gene ontology (GO) "Biological Process" terms of the DETs

Table S7). Of these, only XIST was shared at all time points. Apart from XIST, none of overlapping DETs were expressed from the X-chromosome. Two transcripts were upregulated in fetal and adult KS (RP11-15H7.2 and RP11-44K6.3). One transcript was downregulated in prepubertal and adult KS (GLI1), and the remaining transcripts showed different directions of regulation between two time points (Fig. 7c).

\section{Discussion}

It is intriguing that an extra X-chromosome in KS patients is associated with loss of germ cells, although the adult testicular phenotype with degeneration and hyalinization of seminiferous tubules does not develop until puberty. It is to be expected that chromosome pairing and segregation during meiosis is impaired with the presence of an extra chromosome. However, since the loss of spermatogonia begins before puberty, even as early as during fetal development, other mechanisms must be involved. To bring new insights into the functional mechanisms that result in germ cell loss and testicular degeneration we analyzed the testicular transcriptome. It was admittedly challenging to obtain informative transcriptome profiles from the archived material. Furthermore, tubular and interstitial compartments of the testis contain many different types of cells. In order to tackle this, we collected all the testicular biopsies from KS men that were available in our archives. However, of the 12 samples available, only 10 could be used for RNAsequencing and only $3 \mathrm{KS}$ samples were found suitable in 

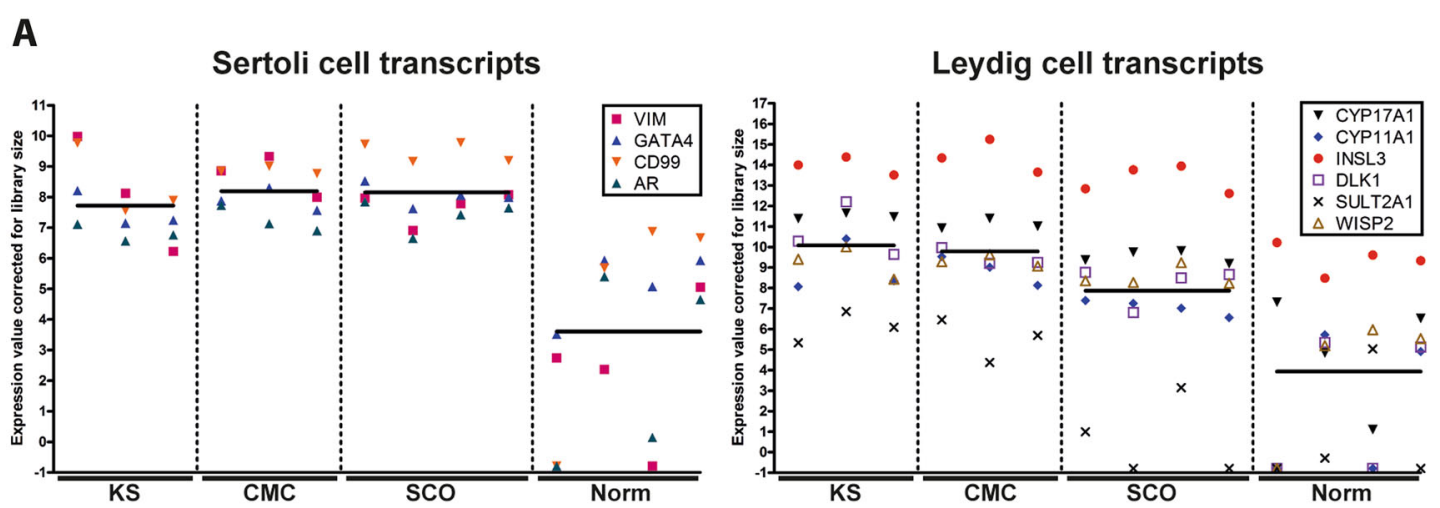

Peritubular and blood vessel transcripts
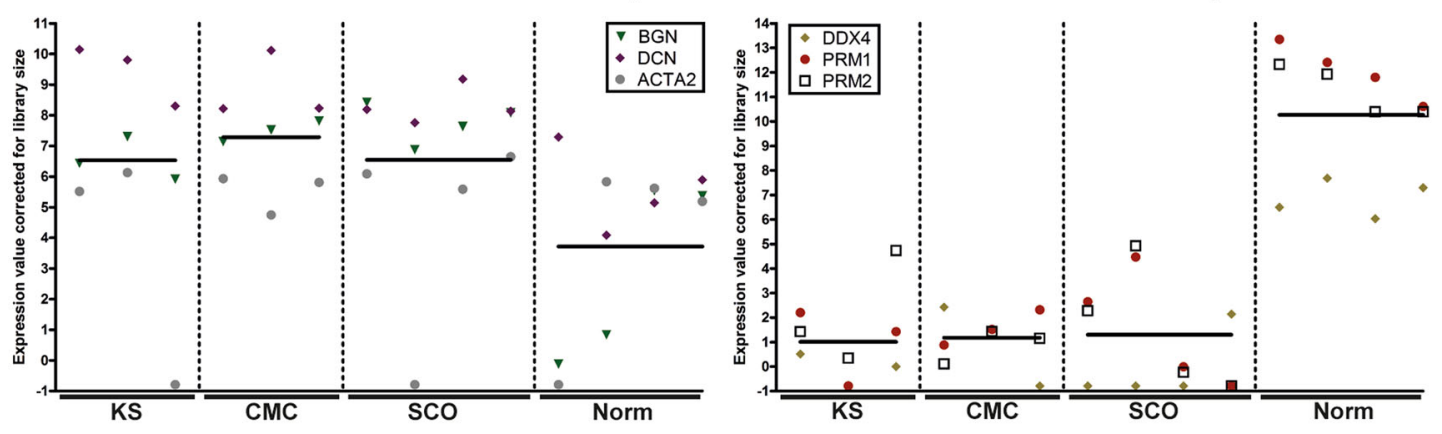

B
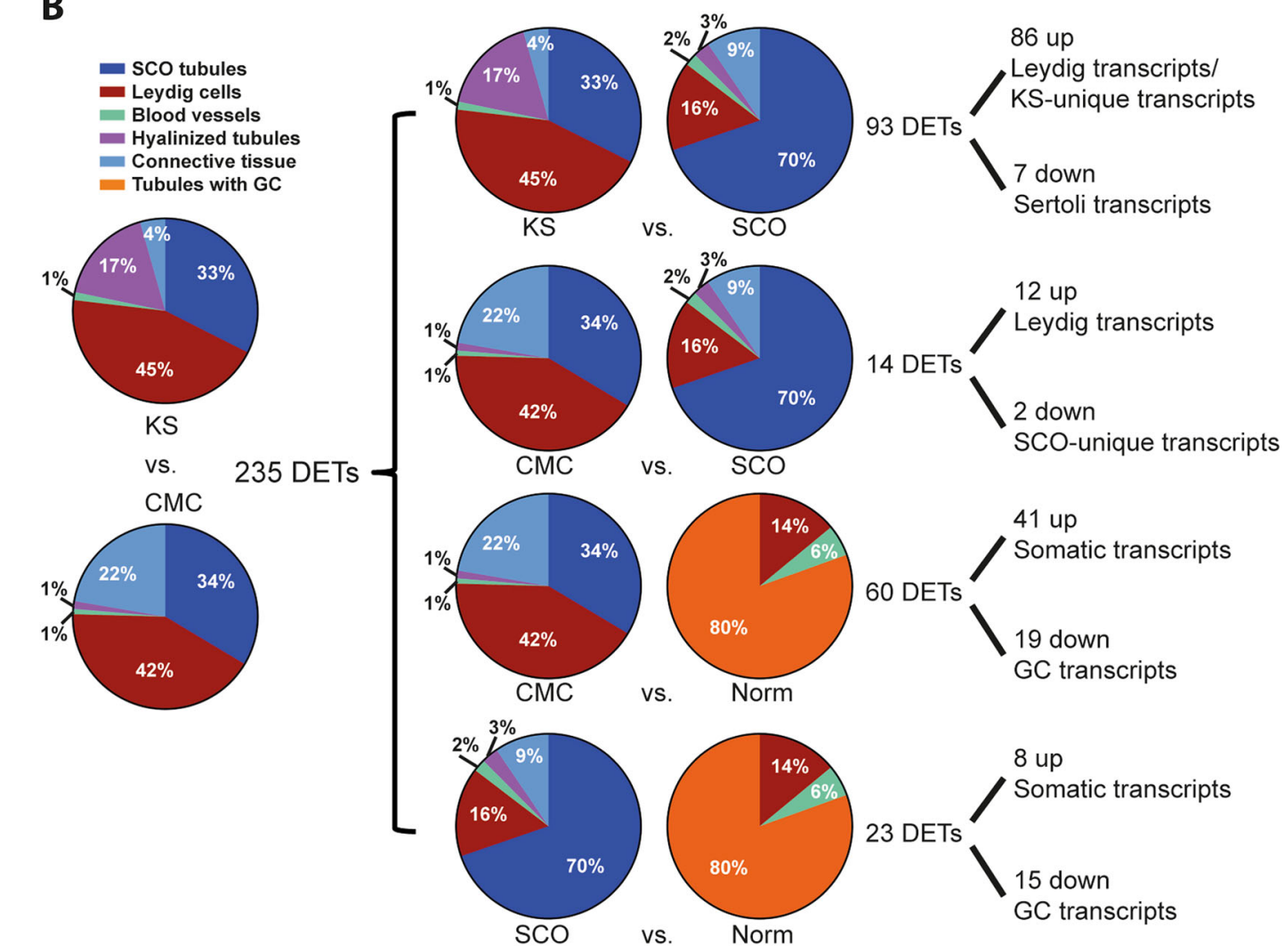

Fig. 5 Depicted cellular origin of the differentially expressed transcripts (DETs). a Known Sertoli, Leydig, peritubular/blood vessel and germ cell
markers expressed in the testis of Klinefelter syndrome (KS), cellularity-matched controls (CMC), Sertoli cell-only (SCO), and normal testes with complete spermatogenesis (Norm). The lines indicate the mean expression values in each group. b Pie charts of average cellularity in each group and resultant predicted cellular origin of the overlapping DETs taking into account the amounts of cellularity transcripts in (a) 


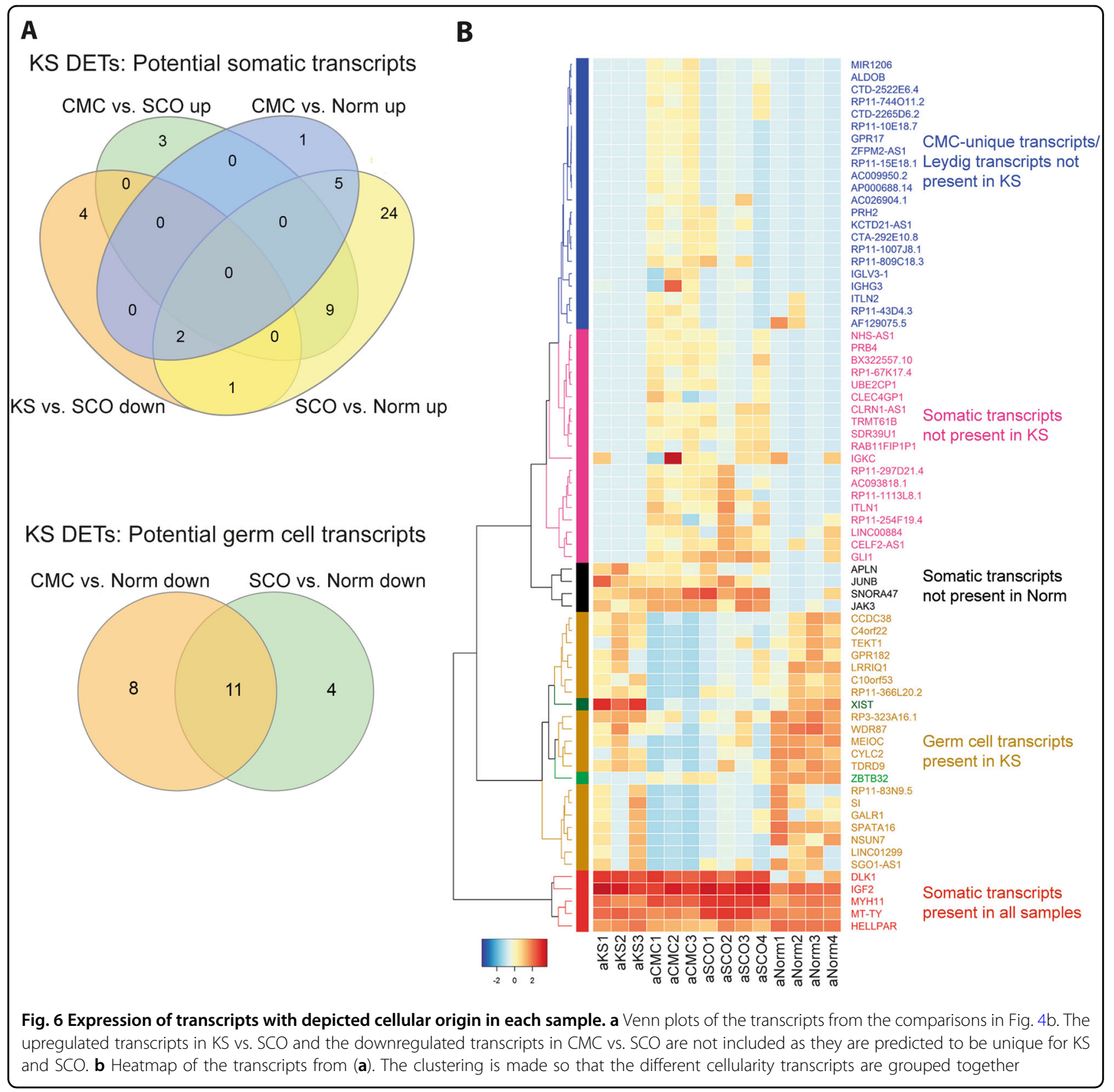

the analysis. Moreover, we did not have statistical power to correct for multiple testing, which is normal practice in transcriptome analysis. Instead, we used an un-adjusted $p$ value and identified 235 DETs potentially involved in the pathogenesis of the KS testicular phenotype.

One of the interesting DETs was $D A C H 2$, with a potential function in "reproduction" and "development" according to the GO analysis. The potential involvement of DACH2 in the testicular phenotype of KS men is supported by two studies. One study showed that Dach2 was expressed in the fetal mouse testis, but not in the ovary, with pronounced expression in interstitial cells. Interestingly, the fetal gonadal expression of Dach2 was lost in the Wnt-4 knock-out testis ${ }^{28}$. Another study identified an association between several rare mutations in the $D A C H 2$ gene and premature ovarian failure, which is characterized by an early loss of germ cells ${ }^{29}$. We validated the protein expression of $\mathrm{DACH} 2$ in the adult KS testis, and observed an interesting staining pattern. DACH2 was specifically expressed in a subset of Sertoli cell nuclei in the KS testis, which was not observed in the CMC. This indicates that DACH2 is a marker of immature Sertoli cells in the adult KS testis, which was supported by identification of DACH2 in fetal Sertoli cells and by the majority of DACH2-positive Sertoli cells in the adult KS testis being positive for 


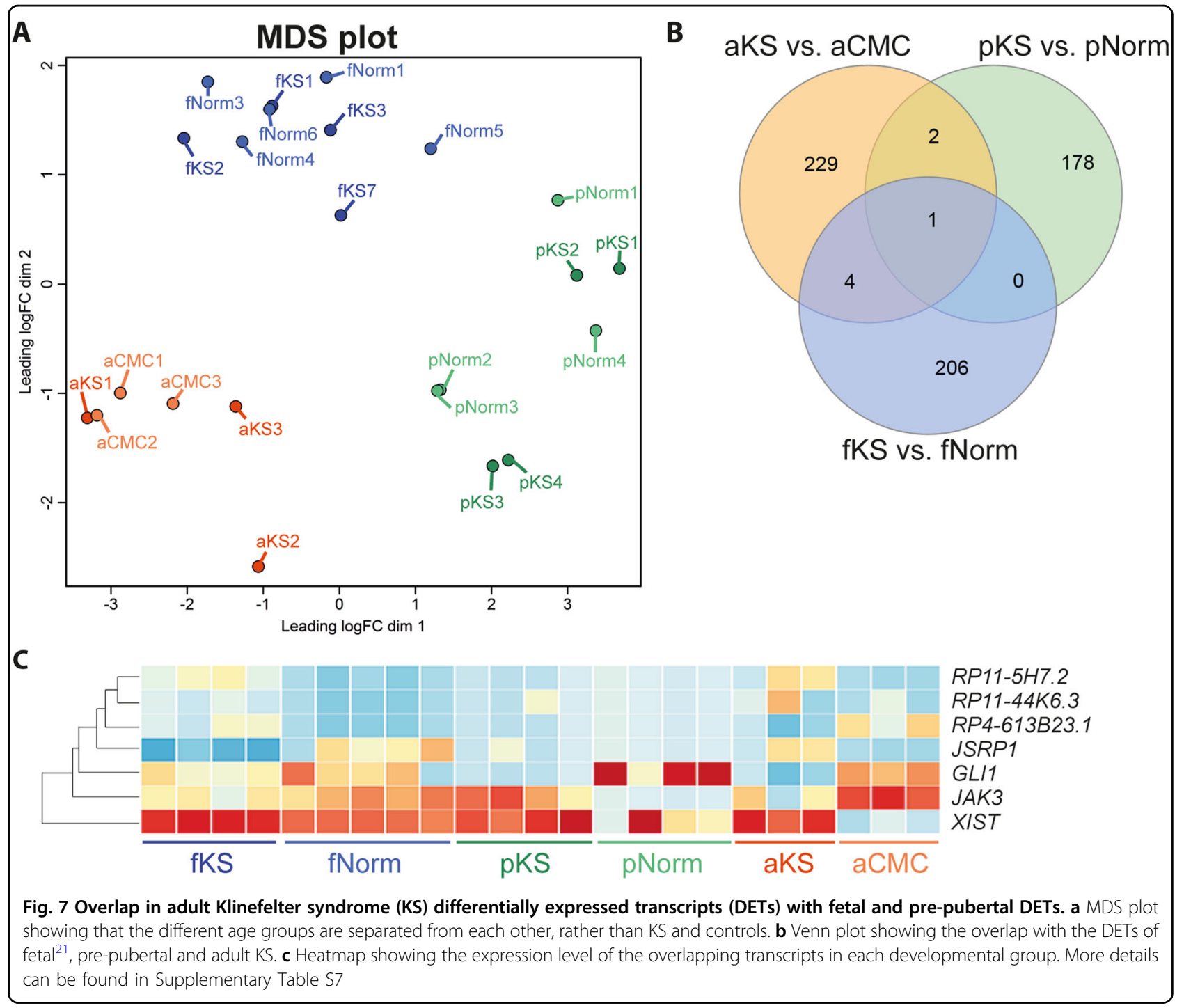

AMH. Further studies are needed to corroborate this finding.

Interestingly, FAM9A, also appeared to be linked to the somatic cell maturation stage. FAM9A was expressed in a subset of Leydig cells, which appeared to be less mature than normal adult Leydig cells, which express INSL ${ }^{26}$, and FAM9A was also identified in fetal Leydig cells. FAM9A was, in addition, expressed in all nuclei of Sertoli cells, but not at the fetal stage. The difference in the subcellular localization between Leydig- and Sertoli cells indicates that FAM9A has different functions in the two cell types. Nevertheless, both DACH2 and FAM9A seem to mark less mature somatic cell types in the KS confirming previous observations of immature Sertoli- and Leydig cells in the adult KS testis ${ }^{25,26}$. This hypothesis is further supported by the enrichment of GO terms, which revealed overrepresentation of terms related to "reproduction", "development", and "response to hormone".
Dysregulation of these pathways in general fits very well with the KS phenotype.

Another interesting upregulated KS transcript was PNMA5, which recently was shown to induce apoptosis ${ }^{30}$. According to the GTex portal (http://www.gtexportal.org/ home/gene/PNMA5, accessed 7 Sept 2017). and the Human Protein Atlas (http://www.proteinatlas.org/ ENSG00000198883-PNMA5/tissue, accessed 7 Sept 2017), PNMA is expressed exclusively in the testis and brain. Yet another pro-apoptotic protein upregulated in the KS samples was AIFM3 ${ }^{31}$. Increased apoptosis could be a very relevant mechanism responsible for degeneration of the KS testis but when KS samples were stained with cleaved PARP (cPARP), a known marker of apoptotic cells, we only observed very few positively stained cells (data not shown).

When we compared our 235 KS DETs with previous transcriptome studies of KS samples, the overlap 
consisted of only 12 transcripts. Three DETs, were identified in studies performed on peripheral blood ${ }^{13-16}$ and the remaining nine were identified in studies performed on adult testis biopsies ${ }^{22,23}$. Since the control used in the testicular studies ${ }^{22,23}$ included complete spermatogenesis, the overlap with our study, using $\mathrm{CMC}$, was expected to be limited. Moreover, the microarray approach used in these studies would not detect all types of transcripts (e.g., lncRNAs) contrary to the RNAsequencing approach we used. In line with our previous study of fetal KS samples ${ }^{20}$, we indeed observed an enrichment of lincRNAs among the upregulated transcripts in the adult KS testis. This indicates that dysregulation of lincRNAs may play a central role in the KS testis.

Among the protein-coding DETs, three overlapped with previous studies; DLK1, FAM9A and FBOXO43, which all have been described previously in relation to testicular function. In the mouse, Fbxo43 is expressed solely in the testis and ovary ${ }^{32}$, and was shown to be involved in meiosis I progression in rodent spermatocytes ${ }^{33,34}$. DLK1 is a marker of immature Leydig cells ${ }^{26}$ and its upregulation detected in the previous study $y^{23}$ and in our present study is consistent with the presence of more immature Leydig cells in the KS testis. In line with this, expression of $F A M 9 A$, which has been shown to be testis-specific ${ }^{35}$, was also found in less mature Leydig cells in our present study. Taken together, these results indicate that the somatic compartment in the adult KS testis is less mature than that in CMC.

When comparing the KS DETs in fetal, pre-pubertal and adult testes, the identified overlap was limited, indicating that different mechanisms are responsible for the testicular architecture and cellular composition at the different developmental stages. We did see an overlap in dysregulation of JAK3 and GLI1. JAK3 was upregulated in the pre-pubertal samples but downregulated in the adult samples, and GLI1 was downregulated both in prepubertal and adult samples. A relationship between GLI1 and JAK3 was identified in acute T-cell lymphoblastic leukemia, where their expression levels showed a positive correlation $^{36}$. As GLI1 is a component of Hedgehog signaling, which regulates development and cancer progression ${ }^{37}$, downregulation of this transcript in both pre-pubertal and adult samples, as well as downregulation of JAK3 in the adult samples, may indicate a disturbance in the development of the KS testis. Indeed, Hedgehog signaling within the primary cilia of Sertoli cells from adult KS samples has previously been described to be different to that of Sertoli cells in tubules with ongoing spermatogenesis ${ }^{38}$. In our previous study of the fetal KS testis transcriptome ${ }^{20}$, we found enrichment of $\mathrm{X}$-chromosomal DETs, which we could not recapitulate in the adult (or pre-pubertal) KS testis. This indicates that the effect of the extra X-chromosome is more direct in fetal life than in the adult. The adult KS testis consequently seems a consequence of the disturbed fetal gonadal development caused directly by the extra Xchromosome.

Based on our results we hypothesize that the overexpressed genes from the extra X-chromosome in patients with KS are directly causing changes in the testicular transcriptome primarily in fetal life, leading to the initiation of the germ cell loss, gonadal dysgenesis, and subsequent failure of the differentiation of the somatic cells, which consequently appears immature in the adult KS testis. Clearly our study was limited by lack of power due to compromised quality of the isolated RNA. Hopefully, technical advancements in the field will allow future studies to isolate RNA of better quality. Future studies should also focus on including more samples, as the individual variation in testicular development besides variations in cellularity is quite substantial. The present study nevertheless provided reference data for further mechanistic studies, and could also have some clinical implications concerning management of patients with KS. Our results indicate that intervention by supporting or stimulating development of the testicular somatic niche already during childhood could increase chances of fertility in adulthood. Before puberty, many boys with KS still have germ cells in the testis that with proper support by somatic cells might enter spermatogenesis. Such interventions, however, need to be investigated before they can be considered.

In conclusion, we were able to analyze the transcriptome of a total of 24 adult KS testis and controls. Enrichment analysis and protein expression patterns showed disturbed maturation of Sertoli- and Leydig cells. We identified enrichment of lincRNAs, but not of Xchromosomal transcripts, indicating an indirect effect of the supernumerary $\mathrm{X}$-chromosome. Furthermore, the adult testicular KS transcriptome showed a very limited overlap with that of pre-pubertal and fetal KS testis, indicating that distinct processes occur at these stages of development. Our study indicates that Sertoli- and Leydig cells in the testis of adult men with KS are immature and that impairment of testicular function has its origin early in development.

\section{Materials and methods Tissue samples}

The use of testicular tissue for this project was approved by a regional medical and research ethics committee (permit no. H-2-2014-103) and the Danish Data Protection Agency (no. 2012-58-0004, local no. 30-1482, I-Suite 03696). The testis samples were collected between 1976 and 2014. Following surgical excision, the samples were immediately fixed in different fixatives, dehydrated, and 
embedded in paraffin (Table 1). The blocks were stored either at $+4{ }^{\circ} \mathrm{C}$ or $-20^{\circ} \mathrm{C}$ until sectioning.

\section{Immunohistochemistry (IHC)}

Details on antibodies and detection reagents are shown in Supplementary Table S8.

IHC staining with anti-MAGE-A4 and anti-cPARP antibodies was performed essentially as described before $^{39}$. For MAGE-A4, staining was performed on testes from ten adult men with KS, five with CMC testicular histology, five with Sertoli-cell-only (SCO), and four with full spermatogenesis (Table 1). In brief, paraffinembedded sections were deparaffinized and rehydrated. Antigen retrieval was accomplished by microwaving the sections for $15 \mathrm{~min}$ in unmasking buffer. Then sections were incubated with $2 \%$ non-immune goat serum (Zymed Histostain Kit, Life Technologies, CA, USA) to minimize cross-reactivity. The primary antibody was added and incubated overnight at $+4{ }^{\circ} \mathrm{C}$, and then the sections were incubated with biotinylated goat anti-mouse IgG (MAGEA4) or biotinylated goat-anti-rabbit (cPARP), before a peroxidase-conjugated streptavidin complex was used as a tertiary layer (Zymed Histostain Kit). Visualization was performed with amino ethyl carbasole (Zymed Histostain Kit).

Staining with anti-DACH2 and anti-FAM9A were performed on testes from two adult men with KS, three with full spermatogenesis and three with CMC testicular histology, and staining with anti-AMH was performed on testes from two adult men with KS (Supplementary Table S9), essentially as described before ${ }^{40}$. In brief, sections were subjected to pressure cooking-induced antigen retrieval in unmasking buffer at $110^{\circ} \mathrm{C}$ for $30 \mathrm{~min}$. Unspecific binding was blocked with $0.5 \%$ skimmed milk in TBS. Sections were incubated overnight with primary antibody diluted in skimmed milk at $+4{ }^{\circ} \mathrm{C}$. Horse antirabbit IgG HRP (ImmPRESS detection kit, Vector laboratories, CA, USA) was applied for $30 \mathrm{~min}$ followed by development with diaminobenzidin DAB (ImmPress detection kit) and nuclear counterstaining with Mayer's Hematoxylin. Control sections with omission of primary antibody were all negative (data not shown).

\section{Double immunofluorescence}

Details of antibodies and detection reagents are shown in Supplementary Table S8. Staining was performed on a testis sample from an adult man with KS (Supplementary Table S9) essentially as described before ${ }^{40}$. In brief, following pressure cooking treatment as described for IHC, the section was incubated with horse serum diluted in PBS containing 5\% (w/v) BSA. The anti-FAM9A antibody was applied and the slides were left overnight at $+4{ }^{\circ} \mathrm{C}$ and $1 \mathrm{~h}$ at room temperature. The section was incubated with peroxidase-conjugated chicken anti-rabbit secondary antibody (Sigma-Aldrich, MO, USA) for $30 \mathrm{~min}$ followed by incubation with Tyramide Cy3 (Perkin Elmer, MA, USA) for $10 \mathrm{~min}$. Antigen retrieval with pressure cooker, blockade with horse serum, and incubation with primary antibody was repeated for the anti-INSL3 antibody. The section was then incubated with peroxidase-conjugated chicken anti-rabbit secondary antibody followed by incubation with Tyramide Fluorescien (Perkin Elmer) for 10 min. Finally, the section was counterstained with DAPI (Sigma-Aldrich). The section was imaged with an Olympus BX61 microscope, captured using the Cell Sense Dimensions V1.6 software (Olympus Ltd., Ballerup, Denmark) and processed in Adobe Photoshop 6.0.

\section{Quantification of cellularity}

Adobe Photoshop CC 2014 (Adobe Systems Inc, CA, USA) was used to assess the cellularity of each sample. The area of tubules with germ cells was evaluated on the basis of the MAGE-A4-staining and was quantified with the "lasso" tool. SCO tubules, areas of Leydig cells, blood vessels, hyalinized tubules and connective tissue were identified by visual inspection also measured with the "lasso" tool.

\section{Tissue sectioning}

Fixed, paraffin-embedded tissue was sectioned on a microtome at a thickness of $10 \mu \mathrm{m}$ and placed in Eppendorf tubes at $+4{ }^{\circ} \mathrm{C}$ until RNA extraction. A total of $20-50 \mu \mathrm{m}$ of tissue was used. Serial sections of each tissue were collected before and after the collection on membranes and stored at $-20^{\circ} \mathrm{C}$ for subsequent histological assessment of cellularity.

\section{RNA extraction}

Total RNA was extracted using the Recover All Total Nucleic Acid Isolation Kit for FFPE (Thermo Fisher Scientific, MS, USA) according to the manufacturer's recommendations. RNA was eluted in LoBind tubes using nuclease-free water. To assess the quantity and quality of the samples, the Qubit RNA HS Assay Kit (Thermo Fisher Scientific) and the Agilent RNA 6000 Pico Kit (Agilent Technologies, CA, USA), respectively, were used.

\section{Library preparation}

The Ovation Human FFPE RNA-Seq Multiplex Systems (NuGEN, Leek, Netherlands) were used for library preparation from 150-200 ng of total RNA according to the manufacturer's recommendations with the following modifications: The cDNA samples were fragmented to yield sizes of approximately $150 \mathrm{bp}$ using the Covaris E210 sonicator (Covaris Ltd., Brighton, UK). For the final library amplification, 20 cycles of amplification were used. An extra DNA purification was performed with a 1.4 times overload of Agencourt AMPure XP beads (Beckman 
Coulter, IN, USA) to get rid of adapters and primers. Samples were pooled in groups of five with equal amount of each. We performed $150 \mathrm{nt}$ paired-end read sequencing on the Illumina HiSeq 2000 sequencing platform (Illumina, CA, USA).

\section{Analysis of RNA-sequencing data}

Reads were demultiplexed using a custom perl script and adapter sequences trimmed using AdapterRemoval $\mathrm{v} 1.5^{41}$. Reads were mapped to the human reference genome (GRCh38) in which the pseudoautosomal region (PAR) on the Y-chromosome (PARY; chrY:100012781479, chrY:56887903-57217415) were masked using STAR v. $2.5 .2 \mathrm{~b}^{42}$. Read counts were summarized using HTseq v. 0.6.0 ${ }^{43}$.

Data were analyzed in the R software v. 3.3 .2 (http:// www.r-project.org). Briefly, the count data was loaded into the limma/edge $\mathrm{package} \mathrm{e}^{44,45}$ and heatmaps were generated with the package gplots ${ }^{46}$ using the heatmap. 2 function with the "complete" hierarchical clustering method. Based on this analysis, seven KS samples and two CMC were excluded (Fig. 1a and Supplementary Fig. S1). To avoid accidental findings, we decided to include only transcripts with at least 1 count per million (CPM) in at least two samples in the overexpressing group. This left a total of 22,020 transcripts in the analysis. In Table 1, the library sizes are shown for all samples.

The $R$ package limma ${ }^{47}$ was used to identify DETs and fold changes were calculated as the mean expression values for the two groups of interest and then further $\log _{2}$-transformed. A $p$-value $<0.01$ and a $\log _{2}$ fold change $(\log F C) \geq 1.5$ or $\leq 1.5$ was used as cutoff. Enrichment analyses were performed using mroast and camera functions. XCI status was acquired from ref. ${ }^{24}$ and GENCODE transcript biotypes were obtained from Ensembl using the biomaRt package in $\mathrm{R}^{48,49}$. A hypergeometric test using the phyper function in $\mathrm{R}$ was used to test for enrichment of transcript biotypes. Expression of known Sertoli, Leydig, germ cell, and peritubular/blood cell markers in KS, CMC, SCO, and Norm were plotted with GraphPad Prism version 4 (GraphPad Software Inc, CA, USA).

DETs were identified between $\mathrm{KS}, \mathrm{CMC}, \mathrm{SCO}$, and Norm as performed with the KS vs. CMC, and the overlap was identified in $\mathrm{R}$. The relative expression level of transcripts that overlapped was visualized with the heatmap. 2 function using the "manhattan" distance matrix and the "ward.D2" hierarchical clustering method. The overlap was visualized using the interactive venn website (http:// www.interactivenn.net/, accessed 2 Sept 2017). Overrepresentation of GO terms ${ }^{50}$ (the "Biological Process" terms) was tested using the DAVID Bioinformatics Resources $^{51,52}$.
Data has been deposited in the GEO public database with the accession number GSE103905.

\section{Acknowledgements}

We thank Ana Ricci Nielsen for technical assistance with tissue sectioning and Camilla Tang Thomsen for technical assistance with RNA extraction. This work was supported by the Danish Council for Independent Research | Medical

Sciences [grant number DFF-4004-00412 to ERDM] and the Innovation Fund Denmark [grant number 14-2013-4 to K.A.].

\section{Author details}

'Department of Growth and Reproduction, Copenhagen University Hospital (Rigshospitalet), Copenhagen, Denmark. ${ }^{2}$ DTU Multi-Assay Core, DTU

Bioinformatics, Technical University of Denmark, Kongens Lyngby, Denmark.

${ }^{3}$ Translational Disease Systems Biology Group, Novo Nordisk Foundation for

Protein Research, University of Copenhagen, Copenhagen, Denmark.

${ }^{4}$ Bioinformatics Research Center, Aarhus University, Aarhus, Denmark

Conflict of interest

The authors declare that they have no conflict of interest.

\section{Publisher's note}

Springer Nature remains neutral with regard to jurisdictional claims in published maps and institutional affiliations.

Supplementary Information accompanies this paper at https://doi.org/ 10.1038/s41419-018-0671-1.

Received: 8 February 2018 Revised: 21 April 2018 Accepted: 3 May 2018 Published online: 22 May 2018

\section{References}

1. Bojesen, A., Juul, S. \& Gravholt, C. H. Prenatal and postnatal prevalence of Klinefelter syndrome: a national registry study. J. Clin. Endocrinol. Metab. 88, 622-626 (2003).

2. Aksglaede, L., Skakkebaek, N. E., Almstrup, K. \& Juul, A. Clinical and biological parameters in 166 boys, adolescents and adults with nonmosaic Klinefelter syndrome: a Copenhagen experience. Acta Paediatr. 100, 793-806 (2011).

3. Bonomi, M. et al. Klinefelter syndrome (KS): genetics, clinical phenotype and hypogonadism. J. Endocrinol. Invest. 40, 123-134 (2017).

4. Chang, S., Skakkebaek, A. \& Gravholt, C. H. Klinefelter syndrome and medical treatment: hypogonadism and beyond. Horm. (Athens) 14, 531-548 (2015).

5. Nieschlag, E. et al. The Klinefelter syndrome: current management and research challenges. Andrology 4, 545-549 (2016).

6. Aksglaede, L. et al. Natural history of seminiferous tubule degeneration in Klinefelter syndrome. Hum. Reprod. Update 12, 39-48 (2006).

7. Blanco, J., Egozcue, J. \& Vidal, F. Meiotic behaviour of the sex chromosomes in three patients with sex chromosome anomalies (47,XXY, mosaic 46,XY/47,XXY and 47,XYY) assessed by fluorescence in-situ hybridization. Hum. Reprod. 16, 887-892 (2001).

8. Garcia-Quevedo, L. et al. Hidden mosaicism in patients with Klinefelter's syndrome: implications for genetic reproductive counselling. Hum. Reprod. 26, 3486-3493 (2011).

9. Sciurano, R. B. et al. Focal spermatogenesis originates in euploid germ cells in classical Klinefelter patients. Hum. Reprod. 24, 2353-2360 (2009).

10. Yamamoto, Y. et al. Morphometric and cytogenetic characteristics of testicular germ cells and Sertoli cell secretory function in men with non-mosaic Klinefelter's syndrome. Hum. Reprod. 17, 886-896 (2002).

11. Corona, G. et al. Sperm recovery and ICSI outcomes in Klinefelter syndrome: a systematic review and meta-analysis. Hum. Reprod. Update 23, 265-275 (2017).

12. Ottesen, A. M. et al. Increased number of sex chromosomes affects height in a nonlinear fashion: a study of 305 patients with sex chromosome aneuploidy. Am. J. Med Genet Part A 152A, 1206-1212 (2010).

13. Belling, $K$. et al. Klinefelter syndrome comorbidities linked to increased $X$ chromosome gene dosage and altered protein interactome activity. Hum. Mol. Genet. 26, 1219-1229 (2017). 
14. Huang, J. et al. Global transcriptome analysis of peripheral blood identifies the most significantly down-regulated genes associated with metabolism regulation in Klinefelter syndrome. Mol. Reprod. Dev. 82, 17-25 (2015).

15. Zitzmann, M. et al. Gene expression patterns in relation to the clinical phenotype in Klinefelter syndrome. J. Clin. Endocrinol. Metab. 100, E518-E523 (2015).

16. Vawter, M. P., Harvey, P. D. \& Delisi, L. E. Dysregulation of X-linked gene expression in Klinefelter's syndrome and association with verbal cognition. Am. J. Med. Genet. B. Neuropsychiatr. Genet. 144B, 728-734 (2007).

17. Lee, J. T. Gracefully ageing at 50, X-chromosome inactivation becomes a paradigm for RNA and chromatin control. Nat. Rev. Mol. Cell Biol. 12, 815-826 (2011).

18. Kleinheinz, A. \& Schulze, W. Klinefelter's syndrome: new and rapid diagnosis by PCR analysis of XIST gene expression. Andrologia $\mathbf{2 6}$, 127-129 (1994).

19. Wikström, A. M. et al. Klinefelter syndrome in adolescence: onset of puberty is associated with accelerated germ cell depletion. J. Clin. Endocrinol. Metab. 89, 2263-2270 (2004)

20. Winge, S. B. et al. Transcriptome profiling of fetal Klinefelter testis tissue reveals a possible involvement of long non-coding RNAs in gonocyte maturation. Hum. Mol. Genet. 27, 430-439 (2018).

21. Alves, M. G. et al. Testicular lactate content is compromised in men with Klinefelter Syndrome. Mol. Reprod. Dev. 83, 208-216 (2016).

22. D'Aurora, M. et al. Deregulation of sertoli and leydig cells function in patients with Klinefelter syndrome as evidenced by testis transcriptome analysis. BMC Genom. 16, 156 (2015)

23. D'Aurora, M. et al. Testis transcriptome modulation in Klinefelter patients with hypospermatogenesis. Sci. Rep. 7, 45729 (2017).

24. Balaton, B. P., Cotton, A. M. \& Brown, C. J. Derivation of consensus inactivation status for X-linked genes from genome-wide studies. Biol. Sex. Differ. 6, 35 (2015).

25. Skakkebaek, N. E. Two types of tubules containing only Sertoli cells in adults with Klinefelter's syndrome. Nature 223, 643-645 (1969).

26. Lottrup, G. et al. Expression patterns of DLK1 and INSL3 identify stages of Leydig cell differentiation during normal development and in testicular pathologies, including testicular cancer and Klinefelter syndrome. Hum. Reprod. 29, 1637-1650 (2014).

27. Harrow, J. et al. GENCODE: the reference human genome annotation for The ENCODE Project. Genome Res. 22, 1760-1774 (2012).

28. Naillat, F. et al. Identification of the genes regulated by Wnt-4, a critical signal for commitment of the ovary. Exp. Cell Res. 332, 163-178 (2015).

29. Bione, S. et al. Mutation analysis of two candidate genes for premature ovarian failure, DACH2 and POF1B. Hum. Reprod. 19, 2759-2766 (2004).

30. Lee, Y. H., Pang, S. W. Poh, C. L. \& Tan, K. O. Distinct functional domains of PNMA5 mediate protein-protein interaction, nuclear localization, and apoptosis signaling in human cancer cells. J. Cancer Res. Clin. Oncol. 142, 1967-1977 (2016).

31. Xie, Q., Lin, T., Zhang, Y., Zheng, J. \& Bonanno, J. A. Molecular cloning and characterization of a human AlF-like gene with ability to induce apoptosis. J. Biol. Chem. 280, 19673-19681 (2005).

32. Shoji, S. et al. Mammalian Emi2 mediates cytostatic arrest and transduces the signal for meiotic exit via Cdc20. EMBO J. 25, 834-845 (2006).
33. Perrard, M. H., Chassaing, E., Montillet, G., Sabido, O. \& Durand, P. Cytostatic factor proteins are present in male meiotic cells and beta-nerve growth factor increases mos levels in rat late spermatocytes. PLOS ONE 4, e7237 (2009).

34. Gopinathan, L. et al. Emi2 is essential for mouse spermatogenesis. Cell Rep. 20 397-708 (2017).

35. Martinez-Garay, I. et al. A new gene family (FAM9) of low-copy repeats in Xp22.3 expressed exclusively in testis: implications for recombinations in this region. Genomics 80, 259-267 (2002).

36. Dagklis, A. et al. Hedgehog pathway activation in T-cell acute lymphoblastic leukemia predicts response to SMO and GLI1 inhibitors. Blood 128, 2642-2654 (2016).

37. Altaba, A., Mas, C. \& Stecca, B. The Gli code: an information nexus regulating cell fate, stemness and cancer. Trends Cell Biol. 17, 438-447 (2007).

38. Nygaard, M. B., Almstrup, K., Lindbaek, L., Christensen, S. T. \& Svingen, T. Cell context-specific expression of primary cilia in the human testis and ciliary coordination of Hedgehog signalling in mouse Leydig cells. Sci. Rep. 5, 10364 (2015).

39. Blomberg Jensen, $M$. et al. Vitamin D receptor and vitamin D metabolizing enzymes are expressed in the human male reproductive tract. Hum. Reprod. 25, 1303-1311 (2010)

40. Mitchell, R. T. et al. Xenografting of human fetal testis tissue: a new approach to study fetal testis development and germ cell differentiation. Hum. Reprod. 25, 2405-2414 (2010)

41. Lindgreen, S. AdapterRemoval: easy cleaning of next-generation sequencing reads. BMC Res. Notes 5, 337 (2012).

42. Dobin, A. et al. STAR: ultrafast universal RNA-seq aligner. Bioinformatics 29 15-21 (2013).

43. Anders, S., Pyl, P. T. \& Huber, W. HTSeq-a Python framework to work with high-throughput sequencing data. Bioinformatics 31, 166-169 (2015).

44. McCarthy, D. J., Chen, Y. \& Smyth, G. K. Differential expression analysis of multifactor RNA-Seq experiments with respect to biological variation. Nucleic Acids Res. 40, 4288-4297 (2012).

45. Robinson, M. D., McCarthy, D. J. \& Smyth, G. K. edgeR: a Bioconductor package for differential expression analysis of digital gene expression data. Bioinformatics 26, 139-140 (2010).

46. gplots: Various R Programming Tools for Plotting Data. R package version 3.0.1. http://CRAN.R-project.org/package=gplots

47. Ritchie, M. E. et al. Limma powers differential expression analyses for RNAsequencing and microarray studies. Nucl. Acids Res. 43, e47 (2015).

48. Durinck, S. et al. BioMart and bioconductor: a powerful link between biological databases and microarray data analysis. Bioinformatics 21, 3439-3440 (2005).

49. Durinck, S., Spellman, P. T., Birney, E. \& Huber, W. Mapping identifiers for the integration of genomic datasets with the R/Bioconductor package biomaRt. Nat. Protoc. 4, 1184-1191 (2009).

50. Ashburner, M. et al. Gene ontology: tool for the unification of biology. The Gene Ontology Consortium. Nat. Genet. 25, 25-29 (2000).

51. Huang, D. W., Sherman, B. T. \& Lempicki, R. A. Bioinformatics enrichment tools: paths toward the comprehensive functional analysis of large gene lists. NuCl. Acids Res. 37, 1-13 (2009).

52. Huang, D. W., Sherman, B. T. \& Lempicki, R. A. Systematic and integrative analysis of large gene lists using DAVID bioinformatics resources. Nat. Protoc. $\mathbf{4}$, 44-57 (2009). 